Article

\title{
Synthesis and Properties of Annulated 2-(Azaar-2-yl)- and 2,2'-Di(azaar-2-yl)-9,9'-spirobifluorenes
}

\author{
Jing Lu Liang, Hyochang Cha and Yurngdong Jahng* \\ College of Pharmacy, Yeungnam University, Gyeongsan 712-749, Korea \\ * Author to whom correspondence should be addressed; E-Mail: ydjahng@ynu.ac.kr; \\ Tel./Fax: +82-53-810-2821.
}

Received: 5 August 2013; in revised form: 29 October 2013 / Accepted: 30 October 2013 / Published: 5 November 2013

\begin{abstract}
A series of 9,9'-spirobifluorene-derived $N$-heterocycles were prepared by the reactions of 8,9-dihydrospiro(benzo[ $b]$ fluorene-11,9'-fluoren)-6(7H)-one and 8,8',9,9'tetrahydro-11,11'-spirobi(benzo[b]fluorene)-6,6' $\left(7 H, 7^{\prime} H\right)$-dione with a series of 2-aminoarenecarbaldehydes such as 2-aminobenzaldehyde, 2-aminonicotinealdehyde, 1-amino-2naphthaldehyde, and 8-aminoquinoline-7-carbaldehyde. In addition to the absorption maxima based on the parent 9,9'-spirobifluorene skeleton in the 225-234, 239-280, 296-298, and 308-328 $\mathrm{nm}$ regions, the absorptions due to the $\pi-\pi^{*}$ transitions of the heterocycles were observed in the $351-375 \mathrm{~nm}$ region in the UV absorption spectra. All the compounds showed strong photoluminescences in the $390-430 \mathrm{~nm}$ region.
\end{abstract}

Keywords: 9,9'-spirobifluorene; quinoline; 1,8-naphthyridine; benzo[h]quinoline; 1,10-phenanthroline; XRD; photoluminescence

\section{Introduction}

9,9'-Spirobifluorene (1) as well as its derivatives have been of interest due to their characteristic structures [1], of which the early studies were focused not only on the molecular recognition of various $\alpha$-aminoalcohols by 9,9 -spirobifluorene-based crown ethers [2,3] and of monosaccharides by dendritic cleft receptors [4-6], but also on the inclusion of compounds with hydrocarbons [7], carbohydrates [8,9], and $\alpha$-hydroxycarboxylates [10]. However, interest in 9,9'-spirobifluorenes, especially 9,9'-spirobifluorenebased oligomers, as well as polymers has recently switched to applications in molecular electronic devices for light emitting devices [11-16], hybrid porous solid [17] and catalysis [18]. 
Nevertheless, only a few of monomeric 2,2'-di(heteroaryl)-9,9'-spirobifluorenes such as 2,2'-di(benzo[h]quinol-2-yl)-9,9'-spirobifluorene [19], 2,2'-di(1,10-phenanthrol-2-yl)-9,9'-spirobifluorene [20,21], 2,2',7,7'-tetra(benzo[h]quinol-2-yl)-9,9'-spirobifluorene [22], and 2,2'-di-(benzo[b]-1,10-phenanthrolin-2-yl)-9,9'-spirobifluorene [23] have been reported, and even fewer monoazaaryl-9,9'-spirobifluorenes, such as 2-(4-phenylquinol-2-yl)-9,9'-spirobifluorene [24] and a series of 2-(azaar-2-yl)-9,9'-spirobifluorenes [25] were reported. Even though some of these showed promising photophysical properties for OLED applications, no systematic approaches for the preparation or for the examination of their properties of monomeric 9,9'-spirobifluorene-based heteroaromatics have been pursued as yet. Our interest in the preparation and properties of 9,9'-spirobifluorene-derived polydentates [23,25] encouraged us to thus study a series of 3,2"-annulated 2-(azaheteroar-2"-yl)-9,9'-spirobifluorenes and 3,2";3',2"-annulated 2,2'-di(azaheteroar-2"-yl)-9,9'spirobifluorenes.

\section{Results and Discussion}

\subsection{Synthesis}

Synthesis of 9,9'-spirobifluorene-derived $N$-heterocycles was straightforward, as shown in Schemes 1 and 2. Friedländer condensation of $\mathbf{3}$ with a series of $o$-aminoaldehydes $\mathbf{4}$ such as 2-aminobenzaldehyde [26], 2-aminonicotinaldehyde [27], 1-aminonaphthalene-2-carbaldehyde, and 8-aminoquinoline-7-carbaldehyde [28], afforded the corresponding heteroaromatics 5 with a 9,9 '-spirobifluorene skeleton in $76 \%-82 \%$ yields.

Scheme 1. Synthesis of annulated monoazaar-2-yl-9,9'-spirobifluorenes.

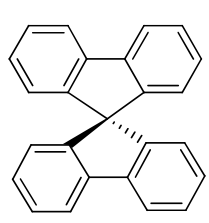

1

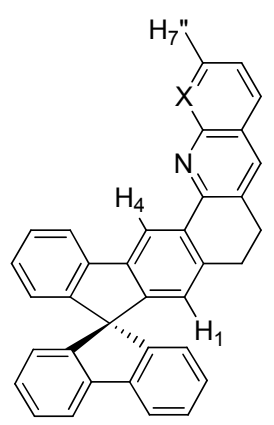

$5 \mathbf{a} \mathrm{X}=\mathrm{CH}$

b $X=N$

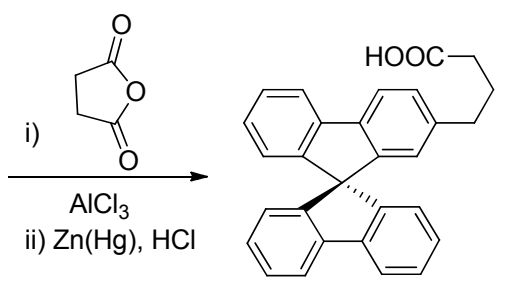

2

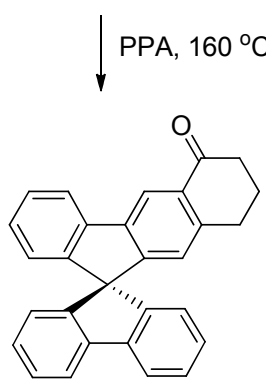

3

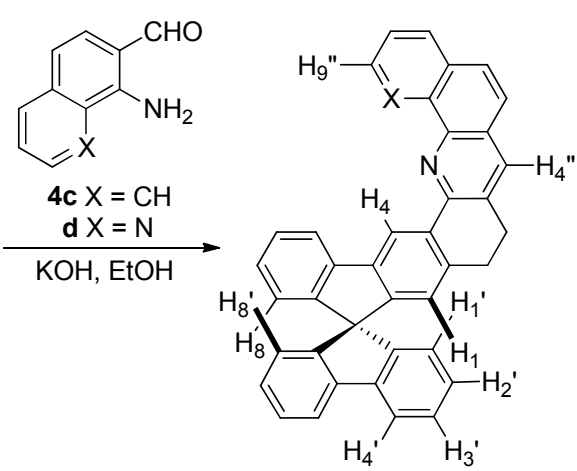

$5 \mathrm{c} \mathrm{X}=\mathrm{CH}$ d $X=N$ 
Scheme 2. Synthesis of annulated 2,2'-di(azaar-2-yl)-9,9'-spirobifluorenes.
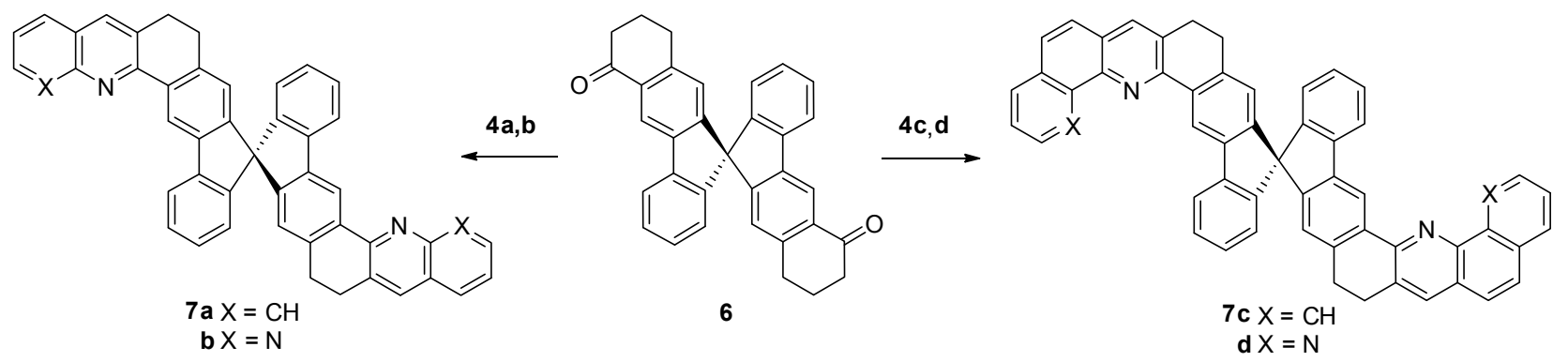

Similarly, the same Friedländer reaction of the diketone 6 [2] with a series of $o$-aminoarenecarbaldehydes afforded the corresponding ligands 7 in $75 \%-82 \%$ yields. The prerequisite ketones 3 and $\mathbf{6}$ were prepared by polyphosphoric acid (PPA)-catalyzed Friedel-Crafts acylation of known 4-[9,9'-spirobi(fluoren)-7-yl]butanoic acid (2) and 4,4'-(9,9'-spirobi[fluorene]-7,7'-diyl)dibutanoic acid [2] in $70 \%$ and $78 \%$ yields, respectively.

\subsection{Structural and Thermal Properties}

The crystallinity of 5 and 7 was accessed by XRD (X-ray diffraction) as shown in Figure 1. All the X-ray diffractograms of the monoheteroaryl systems $\mathbf{5 a , d}$, including $\mathbf{5 b , c}$ (not shown) showed numerous distinctive peaks indicating sharp crystalline nature while those of diheteroaryl systems 7a,d showed a characteristic amorphous halo and were free from any type of crystalline peaks.

Figure 1. X-ray diffractograms of selected compounds 5a, 5d, 7a, and 7d in powder state.

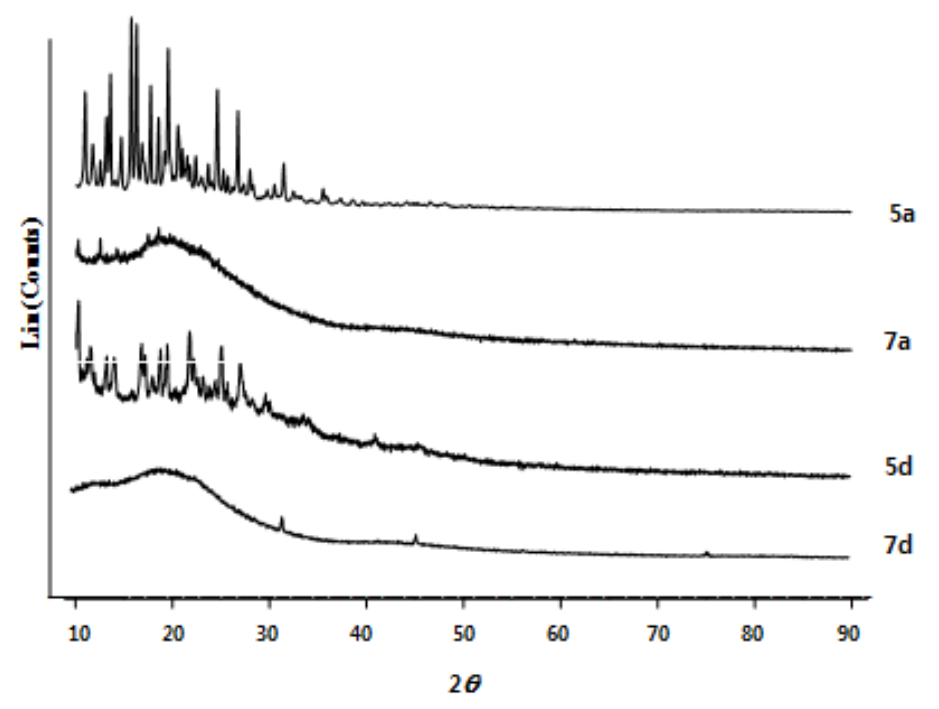

The thermal behaviors of $\mathbf{5}$ and $\mathbf{7}$ were analyzed by differential scanning calorimetry (DSC). All the compounds exhibited a single sharp endothermic peak at the melting points. However, neither glass transition temperatures $(\mathrm{Tg})$ nor crystallization temperature $(\mathrm{Tc})$ were observed for any of the compounds. 


\subsection{Spectroscopic Properties}

2D and DEPT NMR experiments and comparison with the NMR data of the parent 9,9'-spirobifluorene [29] and related compounds reported previously [23,25] allowed us to assign all the proton resonances of the products, which are summarized in Table 1. Some proton resonances were characteristic enough to provide a probe for the suggested structure. The resonances of H4 of the 9,9'spirobifluorene moiety were generally deshielded by the neighboring $N$ in the cavity, and thus resonated in the $\delta$ 9.01-9.38 region, showing significant down-field shifts by $\Delta \delta 1.17-1.54 \mathrm{ppm}$ as compared with the parent 9,9'-spirobifluorene. On the other hand, the H1, H8, H1', and H8' protons of the 9,9'-spirobifluorene moiety were upfield-shifted due to the anisotropic effect of the two neighboring phenyl groups, and thus resonated in the $\delta 6.52-6.87$ range. The H1 resonances of 5 and 7 were upfield-shifted by $\Delta \delta 0.05-0.21 \mathrm{ppm}$, except in $\mathbf{7 c}$, while those of the H8 protons of 7 are slightly downfield-shifted compared to the corresponding proton resonance of the parent 9,9'-spirobifluorene. Additionally, the protons at the peri-position (H7") of 5a and 7a resonated at $\delta 8.21$ and 8.22, respectively, and H10" of the benzo[h]quinoline moiety of $\mathbf{5 c}$ and $\mathbf{7 c}$ resonated at $\delta 9.62$ and 9.63, respectively, as a doublet of doublets $\left({ }^{3} J=7.8 \mathrm{~Hz}\right.$ and $\left.{ }^{4} J=1.0 \mathrm{~Hz}\right)$, evidencing the anisotropic effect of the neighboring pyridine as well as the electronic influence of the lone pair electrons on the central $N$. In addition, the protons adjacent to nitrogen (H7" of $\mathbf{5 b}$ and $\mathbf{7 b}, \mathrm{H} 9 "$ of $\mathbf{5 d}$ and $\mathbf{7 d}$ ) give one of the characteristic chemical shifts ( $\delta 9.08$ and 9.09 for H7" and 9.26 and 9.28 for H9", respectively) as well as relatively small and characteristic ${ }^{3} J$ coupling constants $\left({ }^{3} J=4.3 \mathrm{~Hz}\right)$ [30]. Similar characteristic proton resonances for selected protons were also observed in the ${ }^{1} \mathrm{H}-\mathrm{NMR}$ spectra of 7 .

Table 1. Selected proton resonances for compounds 5 and 7.

\begin{tabular}{ccccccccc}
\hline & H1 $^{\mathbf{a}}$ & $\mathbf{H 4}$ & $\mathbf{H 8}$ & H1' $^{\prime}$ & H7" $^{\prime}$ & H8" $^{\prime}$ & H9" & H10" \\
\hline $\mathbf{1}$ & 7.63 & $7.84[29]$ & - & - & - & - & - & - \\
$\mathbf{5 a}$ & 6.62 & 9.01 & 6.72 & 6.78 & - & 8.21 & - & - \\
$\mathbf{5 b}$ & 6.60 & 9.25 & 6.72 & 6.78 & 9.08 & - & - & - \\
$\mathbf{5 c}$ & 6.52 & 9.22 & 6.72 & 6.78 & - & 7.72 & 7.84 & 9.62 \\
$\mathbf{5 d}$ & 6.62 & 9.34 & 6.71 & 6.78 & 8.25 & 7.64 & 9.26 & - \\
$\mathbf{7 a}$ & 6.67 & 9.05 & 6.79 & - & - & 8.22 & - & - \\
$\mathbf{7 b}$ & 6.69 & 9.28 & 6.80 & 6.69 & 9.09 & - & - & - \\
$\mathbf{7 c}$ & 6.74 & 9.25 & 6.87 & 6.74 & 8.18 & 7.73 & 7.84 & 9.63 \\
$\mathbf{7 d}$ & 6.68 & 9.38 & 6.80 & - & 8.26 & 7.65 & 9.28 & - \\
\hline \multicolumn{7}{c}{ a Numbering pattern and numbering were shown in Scheme 1. }
\end{tabular}

UV absorption spectra of 9,9'-spirobifluorene (1) and its derivatives 5 and $\mathbf{7}$ were obtained in 95\% $\mathrm{EtOH}\left(1.0 \times 10^{-5} \mathrm{M}\right)$ at $298 \mathrm{~K}$ (Figure 2) and results are summarized in Table 2. Five major absorption maxima due to the $\pi-\pi^{*}$ transition were observed in the 225-234, 239-280, 296-298, 308-328, and $351-375 \mathrm{~nm}$ regions. The wavelength of the absorption maximum is highly dependent on the nature of the heterocycles attached to the 9,9'-spirobifluorene core. The systems with benzo $[h]$ quinoline(s) (compounds 5c and 7c), and 1,10-phenanthroline(s) (compounds 5d and 7d) showed additional absorptions in the 351-375 $\mathrm{nm}$ range, which were not observed in the parent 9,9'-spirobifluorene, indicating that these absorptions were from the $\pi-\pi^{*}$ transitions of the heterocycles in the system. Such 
absorptions in compounds with more aromatic rings such as $\mathbf{5 c , d}$ and $\mathbf{7 c}, \mathbf{d}$ were resolved to show two absorptions in the 351-357 and 359-375 $\mathrm{nm}$ regions, respectively, presumably due to the extended delocalization of $\pi$-electrons.

Table 2. UV absorption and fluorescence spectral data of spirobifluorene, $\mathbf{5}$ and $\mathbf{7 .}$

\begin{tabular}{cl|c}
\hline Comp & \multicolumn{1}{c|}{$\lambda_{\max } / \mathbf{n m}\left(\mathbf{1} \times \mathbf{1 0}^{-\mathbf{5}} \mathbf{M}\right.$ in 95\% EtOH, $\left.\mathbf{~ l o g} \boldsymbol{\varepsilon}\right)$} & $\lambda_{\mathrm{em}} / \mathbf{n m}$ \\
\hline $\mathbf{1}$ & $225(4.65) 239(4.40) 272(4.29) 296(3.92) 308(4.11)$ & $388(308)$ \\
$\mathbf{5 a}$ & $213(4.97) 265(5.00) 296(4.53) 308(4.46) 343(\mathrm{sh}, 4.28) 357(4.44)$ & $390(357)$ \\
$\mathbf{5 b}$ & $219(4.99) 258(4.95) 296(4.45) 308(4.38) 326(4.45) 367(4.48)$ & $430(367)$ \\
& $214(4.91) 246(\mathrm{sh}, 4.96) 280(4.70) 293(4.66) 309(4.96) 324(4.31)$ & $392(373)$ \\
$\mathbf{5 c}$ & $355(4.35) 373(4.50)$ & \\
& $212(4.96) 234(4.90) 243(4.93) 252(4.91) 297(4.77) 308(4.63) 351(4.34)$ & $395(367)$ \\
$\mathbf{5 d}$ & $367(4.43)$ & $395(359)$ \\
$\mathbf{7 a}$ & $214(4.90) 265(5.00) 295(4.48) 343(\mathrm{sh}, 4.80) 359(4.63)$ & $395(370)$ \\
$\mathbf{7 b}$ & $216(4.92) 258(4.98) 293(\mathrm{sh}, 4.48) 328(4.40) 370(4.67)$ & $390(357)$ \\
$\mathbf{7 c}$ & $214(4.81) 244(4.95) 283(4.67) 315(4.41) 323(4.40) 357(4.40) 374(4.22)$ & $397(369)$ \\
$\mathbf{7 d}$ & $214(4.84) 242(4.97) 252(4.94) 297(4.82) 352(4.45) 369(4.61)$ & \\
\hline
\end{tabular}

* Number in parenthesis is the excitation wavelength for each compound.

The photoluminescence (PL) of $\mathbf{1}, \mathbf{5}$, and 7 at $298 \mathrm{~K}$ was studied in EtOH $\left(1.0 \times 10^{-5} \mathrm{M}\right)$ to show strong emissions in a 390-430 nm range of, but no correlation between emission maxima and the nature of the heteroaryl skeletons were observed.

Figure 2. UV absorption spectra of 1, 5 and 7 in $95 \% \mathrm{EtOH}\left(1.0 \times 10^{-5} \mathrm{M}\right)$.

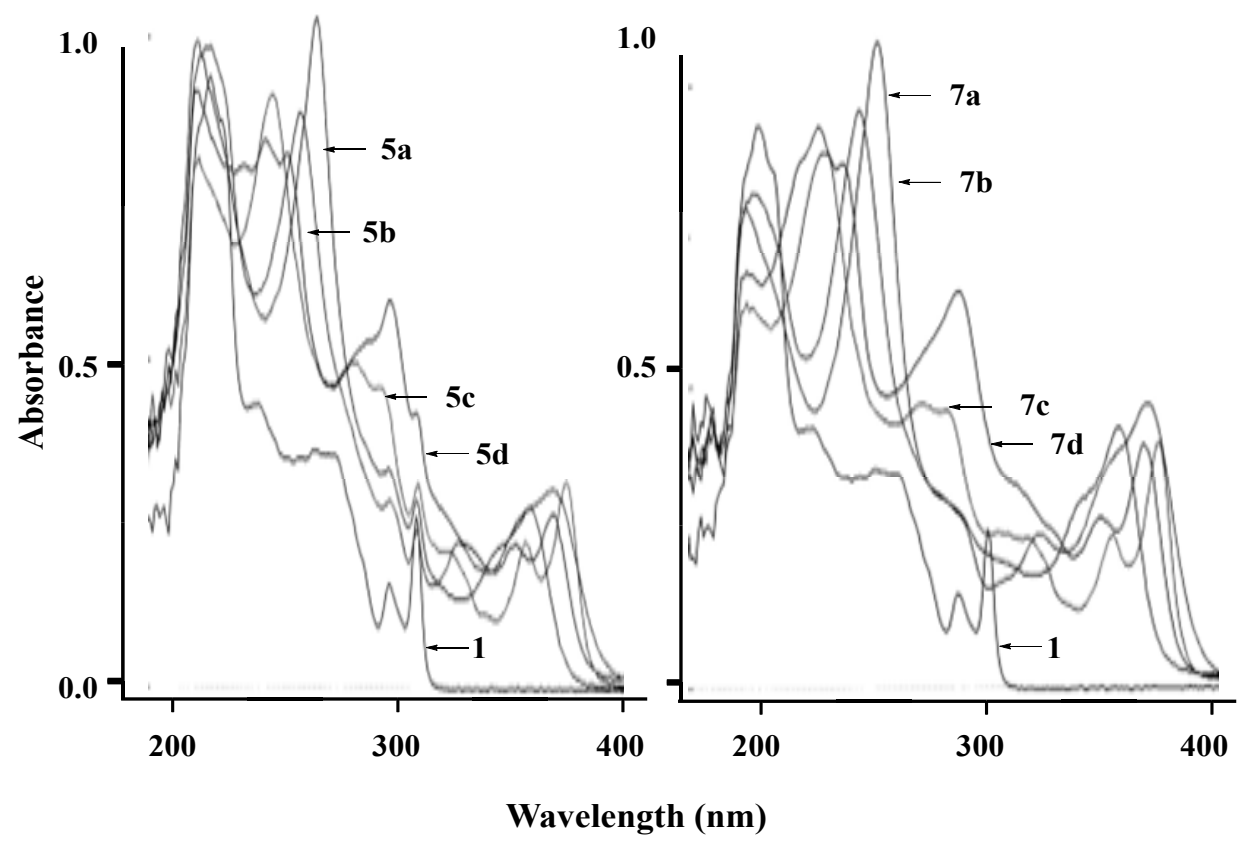




\section{Experimental}

\section{General}

Melting points were determined using a Fischer-Jones melting point apparatus and DSC with tin (mp $232.52{ }^{\circ} \mathrm{C}$ ) as a standard. IR spectra were obtained using a Perkin-Elmer 1330 spectrophotometer. NMR spectra were obtained using a 250 or $600 \mathrm{MHz}$ Bruker-250 spectrometer for ${ }^{1} \mathrm{H}-\mathrm{NMR}$ and 62.5 or $150 \mathrm{MHz}$ for ${ }^{13} \mathrm{C}-\mathrm{NMR}$, respectively, and are reported as parts per million (ppm) from the internal standard tetramethylsilane (TMS) peak. The two-dimensional and DEPT NMR spectra were recorded using Bruker's standard pulse program. UV absorption spectra were recorded on a JASCO-V550 spectrophotometer and emission spectra on a Perkin Elmer LS50B luminescence spectrometer. Electrospray ionization (ESI) mass spectrometry (MS) experiments were performed on a LCQ advantage-trap mass spectrometer (Thermo Finnigan, San Jose, CA, USA). Elemental analyses were taken on a Hewlett-Packard Model 185B elemental analyzer. XRD analysis was performed by X-ray Diffractometer (Model: MPD for bulk, PANalytical, Westborough, MA, USA) with nickel-filtered $\mathrm{CuK} \alpha$ radiatiuon $(30 \mathrm{kV}, 30 \mathrm{~mA})$ at $2 \theta$ angles ranging from $10^{\circ}$ to $90^{\circ}$, a scan speed of $10^{\circ} / \mathrm{min}$ and a time constant of $1 \mathrm{~s}$. Thermal behaviors of the compounds were analysed using differential scanning calorimeter (DSC Q00) with 1 2 mg of sample sealed in alumina in the range of $40-385{ }^{\circ} \mathrm{C}$ increasing temperature in a rate of $10{ }^{\circ} \mathrm{C} / \mathrm{min}$. An empty pan was used as a reference, and the DSC baseline, temperature, and enthalpy was calibrated. A nitrogen flow rate for the each scanning calorimetric run was $50 \mathrm{~mL} / \mathrm{min}$. The starting 4-[9,9'-spirobi(fluoren)-7-yl]butanoic acid (2) [2], $o$-aminoarenecarbaldehydes [26-28], and diketone (6) [2] were prepared by employing previously reported methods. Chemicals and solvents were of commercial reagent grade and used without further purification. The abbreviations $\mathrm{q}, \mathrm{np}, \mathrm{bq}$, and phen were used for quinoline, 1,8-naphthyridine, benzo $[h]$ quinoline and 1,10-phenanthroline, respectively.

8,9-Dihydrospiro(benzo[b]fluorene-11,9'-fluoren)-6(7H)-one (3). A solution of 4-[9,9'-spirobi(fluoren)-7-yl]butanoic acid [2] $(0.30 \mathrm{~g}, 0.75 \mathrm{mmol})$ in PPA $(60 \mathrm{~mL})$ was heated for $40 \mathrm{~min}$ and poured to ice water. The precipitate formed was collected and chromatographed on silica gel eluting with $\mathrm{CH}_{2} \mathrm{Cl}_{2}$ to give pale yellow needles $\left(0.20\right.$ g, 70\%): m.p. $187-189{ }^{\circ} \mathrm{C} .{ }^{1} \mathrm{H}-\mathrm{NMR}\left(\mathrm{CDCl}_{3}, 250 \mathrm{MHz}\right)$ $\delta 8.50$ (s, 1H, H5), 7.84 (m, 3H, H4, H4', H4"), 7.38 (m, 3H, H3, H3', H3"), 7.11 (m, 3H, H2, H2', H2"), 6.72 (m, 3H, H1, H1', H1"), 6.62 (s, H10), 2.74 (t, 2H, $J=6.5 \mathrm{~Hz}), 2.64$ (t, 2H, $J=6.5 \mathrm{~Hz}), 2.04$ (t, 2H, $J=6.5 \mathrm{~Hz}) .{ }^{13} \mathrm{C}-\mathrm{NMR}\left(\mathrm{CDCl}_{3}, 62.5 \mathrm{MHz}\right) \delta 200.23,148.09,148.94,148.86,141.73,141.67,141.21$, $139.91,127.97,127.80,127.65,127.62,127.44,124.06,123.97,123.92,119.92,119.85,119.70,65.90$, $35.07,33.16,26.15$.

6',7'-Dihydrospiro(fluorene-9,9'-fluorneo[2,3-c]acridine) (5a). A mixture of ketone 3 (384 mg, $1.00 \mathrm{mmol}$ ) and 2-aminobenzaldehyde (4a, $133 \mathrm{mg}, 1.10 \mathrm{mmol})$ and in absolute EtOH $(20 \mathrm{~mL})$ containing saturated alcoholic $\mathrm{KOH}(1 \mathrm{~mL})$ was refluxed for $5 \mathrm{~h}$. Upon cooling the reaction mixture, a solid was formed, which was collected as a pure yellow crystalline solid product ( $354 \mathrm{mg}, 80 \%)$ : $\mathrm{mp}$ $313.35{ }^{\circ} \mathrm{C}$. IR ( $\left.\mathrm{KBr}\right)$ v 3056, 1593, 1445, $1429824 \mathrm{~cm}^{-1} .{ }^{1} \mathrm{H}-\mathrm{NMR}\left(\mathrm{CDCl}_{3}, 600 \mathrm{MHz}\right) \delta 9.01(\mathrm{~s}, 1 \mathrm{H}$, H4), 8.21 (d, $J=8.2 \mathrm{~Hz}, 1 \mathrm{H}, \mathrm{H} 8$ of q), 8.05 (d, $J=7.5 \mathrm{~Hz}, 1 \mathrm{H}, \mathrm{H} 5 \mathrm{of} \mathrm{q)}, 7.90$ (s, 1H, H4 of q), 7.85 (d, $2 \mathrm{H}, J=7.8 \mathrm{~Hz}, \mathrm{H} 4{ }^{\prime}$ and $\left.\mathrm{H} 5^{\prime}\right), 7.75(\mathrm{~d}, 1 \mathrm{H}, J=8.0 \mathrm{~Hz}, \mathrm{H} 5), 7.68$ (td, $\left.1 \mathrm{H}, J=8.2,1.5 \mathrm{~Hz}, \mathrm{H} 7 \mathrm{of} \mathrm{q}\right), 7.48$ 
(t, $J=7.8,1.5 \mathrm{~Hz}, \mathrm{H} 6$ of q), $7.40(\mathrm{td}, 1 \mathrm{H}, J=8.0,0.8 \mathrm{~Hz}, \mathrm{H} 6), 7.37\left(\mathrm{td}, 2 \mathrm{H}, J=7.8,1.2 \mathrm{~Hz}, \mathrm{H} 3{ }^{\prime}\right.$ and H6'), $7.14(\mathrm{td}, 1 \mathrm{H}, J=8.0,0.8 \mathrm{~Hz}, \mathrm{H} 7), 7.12(\mathrm{td}, 2 \mathrm{H}, J=7.8,1.2 \mathrm{~Hz}, \mathrm{H} 2$ ' and H7'), 6.78 (d, 2H, $J=7.8 \mathrm{~Hz}$, H1' and H8'), $6.72(\mathrm{~d}, 1 \mathrm{H}, J=7.8 \mathrm{~Hz}, \mathrm{H} 8), 6.62(\mathrm{~s}, 1 \mathrm{H}, \mathrm{H} 1), 3.03(\mathrm{t}, 2 \mathrm{H}, J=6.5 \mathrm{~Hz}), 2.79$ (t, 2H, $J=6.5 \mathrm{~Hz}) .{ }^{13} \mathrm{C}-\mathrm{NMR}\left(\mathrm{CDCl}_{3}, 62.5 \mathrm{MHz}\right) \delta 153.63,150.53,148.92,148.61,147.70,141.80,141.73$, 139.65, 134.76, 133.68, 130.70, 129.36, 127.88 (two C's), 127.82, 127.75, 127.68, 65.89, 28.78, 28.69. MS (ESI): $m / z=470[\mathrm{M}+\mathrm{H}]^{+}$, Anal. Calcd for $\mathrm{C}_{36} \mathrm{H}_{23} \mathrm{~N}$ : C, 92.08; H, 4.94; N, 2.98. Found: C, 90.95; $\mathrm{H}, 4.92 ; \mathrm{N}, 2.90$.

6',7'-Dihydrospiro(fluorene-9,9'-indeno[2',1':6,7]naphtho[1,2-b]-1,8-naphthyridine) (5b). The same procedure described above but with 2-aminonicotinaldehyde (4b) afforded the desired product as pale yellow needles (82\%): m.p. $339.03{ }^{\circ} \mathrm{C}$. ${ }^{1} \mathrm{H}-\mathrm{NMR}\left(\mathrm{CDCl}_{3}, 250 \mathrm{MHz}\right) \delta 9.25(\mathrm{~s}, 1 \mathrm{H}, \mathrm{H} 4), 9.08$ (dd, $J=4.3,2.0 \mathrm{~Hz}, 1 \mathrm{H}, \mathrm{H} 7 \mathrm{of} \mathrm{np}), 8.09$ (dd, $J=8.3,1.5 \mathrm{~Hz}, 1 \mathrm{H}, \mathrm{H} 5 \mathrm{of} \mathrm{np}), 8.04$ (d, $J=8.5 \mathrm{~Hz}, 1 \mathrm{H}, \mathrm{H} 5)$, 7.90 (s, H4 of np), $7.86\left(\mathrm{~d}, 2 \mathrm{H}, J=7.5 \mathrm{~Hz}, \mathrm{H} 4{ }^{\prime}\right.$ and H5'), 7.44-7.34 (m, 4H, H6, H3', H6', H6 of np), $7.11\left(\mathrm{td}, 2 \mathrm{H}, J=7.5,0.8 \mathrm{~Hz}, \mathrm{H} 2^{\prime}\right.$ and H7'), $7.10(\mathrm{dd}, 1 \mathrm{H}, J=7.5,0.8 \mathrm{~Hz}, \mathrm{H} 7), 6.78(\mathrm{~d}, 2 \mathrm{H}$, $J=7.5 \mathrm{~Hz}, \mathrm{H} 1^{\prime}$ and H8'), $6.72(\mathrm{~d}, 1 \mathrm{H}, J=7.5 \mathrm{~Hz}, \mathrm{H} 8), 6.60(\mathrm{~s}, 1 \mathrm{H}, \mathrm{H} 1), 3.06$ (t, 2H, $\left.J=6.7 \mathrm{~Hz}\right), 2.81$ $(\mathrm{t}, 2 \mathrm{H}, J=6.7 \mathrm{~Hz}) .{ }^{13} \mathrm{C}-\mathrm{NMR}\left(\mathrm{CDCl}_{3}, 62.5 \mathrm{MHz}\right) \delta 153.62,150.52,148.92,148.61,147.70,141.80$, 141.73, 141.29, 139.65, 134.58, 133.68, 130.70, 129.36, 128.72, 127.88 (two C's), 1 27.82, 127.74, $127.68,126.95,126.07,124.23,123.86,123.39,120.57,120.00,117.64,65.89,28.78,28.68 . \mathrm{MS}$ (ESI): $m / z=471[\mathrm{M}+\mathrm{H}]^{+}$, Anal. Calcd for $\mathrm{C}_{35} \mathrm{H}_{22} \mathrm{~N}_{2}$ Calcd for $\mathrm{C}_{35} \mathrm{H}_{22} \mathrm{~N}_{2} \cdot \mathrm{H}_{2} \mathrm{O} \mathrm{C}, 86.04 ; \mathrm{H}, 4.95 ; \mathrm{N}$, 5.73. Found: C, 85.59; H, 5.01; N, 5.89.

8,9-Dihydrospiro(benzo[c]fluoreno[3,2-h]acridine-11,9'-fluorene) (5c). The same procedure described above for 5a but with 1-aminonaphthalene-2-carbaldehyde (4c) afforded the desired product as pale yellow crystalline solid (79\%): m.p. $303.65{ }^{\circ} \mathrm{C} .{ }^{1} \mathrm{H}-\mathrm{NMR}\left(\mathrm{CDCl}_{3}, 600 \mathrm{MHz}\right) \delta 9.62(\mathrm{dd}, 1 \mathrm{H}, J=7.8$, $1.0 \mathrm{~Hz}, \mathrm{H} 10$ of bq), 9.22 (s, 1H, H4), 8.14 (d, 1H, J=7.8 Hz, H7 of bq), 7.95 (s, 1H, H4 of bq), 7.93 (d, $1 \mathrm{H}, J=8.4 \mathrm{~Hz}, \mathrm{H} 5), 7.88$ (d, 2H, $J=7.8 \mathrm{~Hz}, \mathrm{H} 4{ }^{\prime}$ and H5'), 7.84 (td, $\left.1 \mathrm{H}, J=7.8,0.6 \mathrm{~Hz}, \mathrm{H} 9 \mathrm{of} \mathrm{bq}\right)$, $7.79(\mathrm{~d}, 1 \mathrm{H}, J=9.0 \mathrm{~Hz}, \mathrm{H} 5 / \mathrm{H} 6$ of bq), 7.72 (td, 1H, $J=7.8,1.2 \mathrm{~Hz}, \mathrm{H} 8$ of bq), 7.66 (d, 1H, $J=9.0 \mathrm{~Hz}$, $\mathrm{H} 6 / \mathrm{H} 5$ of bq), 7.46 (td, $J=7.5,1.0 \mathrm{~Hz}, 1 \mathrm{H}, \mathrm{H} 6), 7.40$ (td, $J=7.5,1.0 \mathrm{~Hz}, 2 \mathrm{H}, \mathrm{H} 3{ }^{\prime}$ and H6'), 7.14 (m, $3 \mathrm{H}, \mathrm{H} 7, \mathrm{H} 2^{\prime}$ and $\left.\mathrm{H}^{\prime}\right), 6.83\left(\mathrm{~d}, J=7.5 \mathrm{~Hz}, 2 \mathrm{H}, \mathrm{H} 1^{\prime}\right.$ and $\left.\mathrm{H} 8{ }^{\prime}\right), 6.76(\mathrm{~d}, 1 \mathrm{H}, J=7.5 \mathrm{~Hz}, \mathrm{H} 8), 6.52(\mathrm{~s}, 1 \mathrm{H}$, $\mathrm{H} 1), 3.09(\mathrm{t}, 2 \mathrm{H}, J=6.5 \mathrm{~Hz}), 2.85(\mathrm{t}, 2 \mathrm{H}, J=6.5 \mathrm{~Hz}) .{ }^{13} \mathrm{C}-\mathrm{NMR}\left(\mathrm{CDCl}_{3}, 62.5 \mathrm{MHz}\right) \delta 151.82,150.21$, $148.94,148.64,145.22,141.88,141.72,141.17,139.30,134.97,134.12,133.50,131.80,130.95$, 127.87 (two C's), 127.83, 127.74 (two C's), 127.68, 127.23, 126.84, 125.71, 125.01, 124.49, 124.24, 123.92, 123.41, 120.38, 120.00, 117.42, 65.86, 28.62, 28.41. MS (ESI): $m / z=520\left[\mathrm{M}+\mathrm{H}^{+}\right]$, Anal. Calcd for $\mathrm{C}_{40} \mathrm{H}_{25} \mathrm{~N} \cdot \mathrm{H}_{2} \mathrm{O}$ : C, 89.36; H, 5.06; N, 2.61. Found: C, 89.21; H, 4.98; N, 2.64.

8',9'-Dihydrospiro(fluorene-11,9'-indeno [2',1":6,7]naphtha[1,2-b]-1,10-phenanthroline) (5d). The same procedure described above for 5a but with 8-aminoquinoline-7-carbaldehyde (4d) afforded the desired product as pale yellow crystalline solids (76\%): m.p. $395.05{ }^{\circ} \mathrm{C} .{ }^{1} \mathrm{H}-\mathrm{NMR}\left(\mathrm{CDCl}_{3}, 250 \mathrm{MHz}\right) \delta$ 9.35 (s, 1H, H4), 9.26 (dd, $J=4.3,1.8 \mathrm{~Hz}, 1 \mathrm{H}, \mathrm{H} 9$ of phen), 8.25 (dd, $J=8.0,1.8 \mathrm{~Hz}, 1 \mathrm{H}, \mathrm{H} 7$ of phen), 8.17 (d, $J=7.5 \mathrm{~Hz}, 1 \mathrm{H}, \mathrm{H} 7$ of phen), 8.00 (s, 1H, H4 of phen), 7.85 (d, $J=7.5 \mathrm{~Hz}, 2 \mathrm{H}, \mathrm{H} 4$ ' and H5'), 7.74 (AB quartet, $\mathrm{H} 5$ and $\mathrm{H} 6$ of phen), $7.64(\mathrm{dd}, J=8.0,4.3 \mathrm{~Hz}, 1 \mathrm{H}, \mathrm{H} 8$ of phen), 7.40 (td, $J=7.5,1.0 \mathrm{~Hz}, 1 \mathrm{H}, \mathrm{H} 6), 7.37\left(\mathrm{td}, J=7.5,1.0 \mathrm{~Hz}, 2 \mathrm{H}, \mathrm{H} 3^{\prime}\right.$ and H6'), $7.12\left(\mathrm{td}, J=7.5,1.0 \mathrm{~Hz}, 2 \mathrm{H}, \mathrm{H} 2^{\prime}\right.$ 
and $\mathrm{H}^{\prime}$ ), $7.10(\mathrm{td}, J=7.5,1.0 \mathrm{~Hz}, 1 \mathrm{H}, \mathrm{H} 7), 6.78\left(\mathrm{~d}, J=7.5 \mathrm{~Hz}, 2 \mathrm{H}, \mathrm{H} 1^{\prime}\right.$ and $\mathrm{H} 8$ '), $6.71(\mathrm{~d}, J=7.5 \mathrm{~Hz}$, $1 \mathrm{H}, \mathrm{H} 8), 6.62(\mathrm{~s}, 1 \mathrm{H}, \mathrm{H} 1), 3.10(\mathrm{dd}, J=7.5,6.3 \mathrm{~Hz}, 2 \mathrm{H}), 2.82(\mathrm{dd}, J=7.5,6.3 \mathrm{~Hz}, 2 \mathrm{H}) .{ }^{13} \mathrm{C}-\mathrm{NMR}$ $\left(\mathrm{CDCl}_{3}, 62.5 \mathrm{MHz}\right) \delta 153.70,150.51,150.16,149.00,148.50,146.36,145.07,142.16,141.94,141.71$, 141.33, 139.20, 136.17, 134.65, 134.34, 132.66, 128.62, 128.13, 127.86 (two C's), 127.70 (two C's), 127.54, 126.29, 125.94, 124.26, 123.69, 123.11, 122.59, 121.04, 119.95, 118.83, 65.89, 28.68, 28.48. MS (ESI): $m / z=521[\mathrm{M}+\mathrm{H}]^{+}$, Anal. Calcd for $\mathrm{C}_{39} \mathrm{H}_{25} \mathrm{~N}_{2} \cdot 0.75 \mathrm{H}_{2} \mathrm{O}: \mathrm{C}, 87.70 ; \mathrm{H}, 4.81 ; \mathrm{N}, 5.24$. Found C, 87.78; H, 4.81; N, 5.53 .

6,6', 7,7'-Tetrahydro-9,9'-spirobi(fluoreno[2,3-c]acridine) (7a). A mixture of ketone 5 (226 mg, $0.50 \mathrm{mmol})$ and 2-aminobenzaldehyde (4a, $133 \mathrm{mg}, 2.20 \mathrm{mmol})$ in absolute EtOH $(20 \mathrm{~mL})$ containing saturated alcoholic $\mathrm{KOH}(1 \mathrm{~mL})$ was refluxed for $5 \mathrm{~h}$. Upon cooling the reaction mixture, the solid was formed, which was collected as a pure pale yellow crystalline solid (82\%): m.p. $334.24{ }^{\circ} \mathrm{C}$. ${ }^{1} \mathrm{H}-\mathrm{NMR}$ $\left.\left(\mathrm{CDCl}_{3}, 300 \mathrm{MHz}\right) \delta 9.05(\mathrm{~s}, 2 \mathrm{H}, \mathrm{H} 4 \text { and } \mathrm{H} 4)^{\prime}\right), 8.22(\mathrm{~d}, 2 \mathrm{H}, J=8.4 \mathrm{~Hz}, \mathrm{H} 8$ of q), 8.08 (d, 2H, $J=7.6$ Hz, H5 of q), 7.91 (s, 2H, H4 of q), 7.75 (d, 2H, $J=8.1 \mathrm{~Hz}, \mathrm{H} 5$ and H5'), 7.71 (td, 2H, $J=7.8,1.4 \mathrm{~Hz}$, $\mathrm{H} 7$ of q), 7.48 (td, 2H, $J=7.8,1.4 \mathrm{~Hz}, \mathrm{H} 6$ of q), 7.42 (td, 2H, $J=8.1,1.5 \mathrm{~Hz}, \mathrm{H} 6$ and H6'), 7.14 (td, $2 \mathrm{H}, J=8.1,1.2 \mathrm{~Hz}, \mathrm{H} 7$ and H7'), 6.79 (d, 2H, $J=7.8 \mathrm{~Hz}, \mathrm{H} 8$ and H8'), 6.67 (s, 2H, H1 and H1'), 3.05 $(\mathrm{t}, 4 \mathrm{H}, J=5.8 \mathrm{~Hz}), 2.81(\mathrm{t}, 4 \mathrm{H}, J=5.8 \mathrm{~Hz}) \cdot{ }^{13} \mathrm{C}-\mathrm{NMR}\left(\mathrm{CDCl}_{3}, 62.5 \mathrm{MHz}\right) \delta 153.62,150.73,148.73$, $147.70,141.81,141.19,139.75,134.63,133.71,130.72,129.35,128.73,127.89,127.75,126.96$, $126.09,124.07,123.59,120.60,117.65,65.85,28.76,28.69$. MS (ESI): $m / z=623[\mathrm{M}+\mathrm{H}]^{+}$, Anal. Calcd for $\mathrm{C}_{47} \mathrm{H}_{30} \mathrm{~N}_{2}$ : C, 90.65; H, 4.86; N, 4.50. Found: C, 89.67; H, 5.03; N, 4.65.

6,6', 7, 7'-Tetrahydro-9,9'-spirobi(indeno[2',1':6,7]naphtho[1,2-b]-1,8-naphthyridine) (7b). The same procedure described above for $\mathbf{7 a}$ but with 2-aminonicotinaldehyde (4b) afforded the desired product as pale yellow crystalline solid (78\%): m.p. $303.658{ }^{\circ} \mathrm{C} .{ }^{1} \mathrm{H}-\mathrm{NMR}\left(\mathrm{CDCl}_{3}, 250 \mathrm{MHz}\right) \delta 9.28(\mathrm{~s}, 2 \mathrm{H}$, H4 and H4'), 9.09 (dd, 2H, $J=4.3,2.0 \mathrm{~Hz}, \mathrm{H} 7$ of np), 8.11 (dd, 2H, $J=7.5,2.0 \mathrm{~Hz}, \mathrm{H} 5 \mathrm{of} n \mathrm{n}$ ), 8.07 (d, $2 \mathrm{H}, J=7.5 \mathrm{~Hz}, \mathrm{H} 5$ and H5'), 7.92 (s, 2H, H4 of np), 7.438 (td, 2H, J= 7.5, 1.0 Hz, H6 of np), 7.437 (td, $2 \mathrm{H}, J=8.1,1.0 \mathrm{~Hz}, \mathrm{H6}$ and H6'), 7.14 (td, 2H, $J=7.5,1.0 \mathrm{~Hz}, \mathrm{H} 7 \mathrm{and} \mathrm{H}^{\prime}$ '), 6.80 (d, 2H, $J=7.5 \mathrm{~Hz}$, $\mathrm{H} 8$ and $\left.\mathrm{H}^{\prime}\right), 6.69$ (s, 2H, H1 and $\left.\mathrm{H} 1^{\prime}\right), 3.10(\mathrm{t}, 4 \mathrm{H}, J=5.8 \mathrm{~Hz}), 2.87(\mathrm{t}, 4 \mathrm{H}, J=5.8 \mathrm{~Hz}) .{ }^{13} \mathrm{C}-\mathrm{NMR}$ $\left(\mathrm{CDCl}_{3}, 62.5 \mathrm{MHz}\right) \delta 156.77,155.86,152.84,151.61,148.62,141.78,141.38,139.79,136.14,134.50$, 134.08, 132.06, 128.15, 127.89, 124.14, 123.59, 122.34, 121.55, 120.75, 118.86, 60.37, 28.56, 28.46. MS (ESI): $m / z=625[\mathrm{M}+\mathrm{H}]^{+}$, Anal. Calcd for $\mathrm{C}_{45} \mathrm{H}_{28} \mathrm{~N}_{4}: \mathrm{C}, 86.51 ; \mathrm{H}, 4.52 ; \mathrm{N}, 8.97$. Found: C, 86.67; H, 4.42; N, 9.05.

8,8',9,9'-Tetrahydro-11,11'-spirobi(benzo[c]fluoreno[3,2-h]acridine) (7c). The same procedure described above for 7a but with 1-aminonaphthalene-2-carbaldehyde (4c) afforded the desired product as pale yellow crystalline solid (75\%): m.p. $395.05{ }^{\circ} \mathrm{C} .{ }^{1} \mathrm{H}-\mathrm{NMR}\left(\mathrm{CDCl}_{3}, 600 \mathrm{MHz}\right) \delta 9.63(\mathrm{dd}, 2 \mathrm{H}$, $J=7.8,1.0 \mathrm{~Hz}, \mathrm{H} 10$ of bq), 9.25 (s, 2H, H4 and H4'), 8.18 (dd, 2H, J= 7.5, $1.0 \mathrm{~Hz}, \mathrm{H} 7 \mathrm{of} \mathrm{bq}$ ), 7.95 (s, 2H, H4 of bq), 7.93 (d, 2H, $J=7.8 \mathrm{~Hz}, \mathrm{H} 5$ and H5'), 7.84 (td, 2H, $J=7.8,1.0 \mathrm{~Hz}, \mathrm{H} 9$ of bq), 7.79 (d, $2 \mathrm{H}, J=8.4 \mathrm{~Hz}, \mathrm{H} 5 / \mathrm{H} 6$ of bq), 7.73 (td, 2H, $J=7.8,1.0 \mathrm{~Hz}, \mathrm{H} 8$ of bq), 7.67 (d, 2H, $J=8.4 \mathrm{~Hz}, \mathrm{H6} / \mathrm{H} 5$ of bq), 7.49 (td, 2H, $J=7.7,1.0 \mathrm{~Hz}, \mathrm{H6}$ and H6'), 7.19 (td, 2H, $J=7.7,1.0 \mathrm{~Hz}, \mathrm{H} 7$ and H7'), 6.87 (d, $2 \mathrm{H}, J=7.7 \mathrm{~Hz}, \mathrm{H} 8$ and $\left.\mathrm{H}^{\prime}\right), 6.74$ (s, 2H, H1 and H1'), 3.10 (t, 4H, $J=5.8 \mathrm{~Hz}$ ), 2.87 (t, 4H, $J=5.8 \mathrm{~Hz}$ ). ${ }^{13} \mathrm{C}-\mathrm{NMR}\left(\mathrm{CDCl}_{3}, 62.5 \mathrm{MHz}\right) \delta 151.84,150.52,148.84,145.26,141.94,141.11,139.42,135.06$, 
134.13, 133.53, 131.84, 130.97, 127.91, 127.83, 127.77 (two C's), 127.25, 126.85, 125.75, 125.03, 124.52, 124.18, 123.64, 120.41, 117.45, 65.87, 28.66, 28.44. MS (ESI): $m / z=723[\mathrm{M}+\mathrm{H}]^{+}$, Anal. Calcd for $\mathrm{C}_{55} \mathrm{H}_{34} \mathrm{~N}_{4} \cdot \mathrm{H}_{2} \mathrm{O}$ : C, 89.16; H, 4.90; N, 3.78. Found: C, 89.67; H, 4.92; N, 3.91.

8,8',9,9'-Tetrahydro-11,11'-spirobi(indeno[2',1':6,7]naphtho[1,2-b]-1,10-phenanthroline) (7d). The same procedure described above for $7 \mathbf{a}$ but with 8 -aminoquinoline-7-carbaldehyde (4d) afforded the desired product as pale yellow crystalline solid (80\%): m.p. $266.77{ }^{\circ} \mathrm{C} .{ }^{1} \mathrm{H}-\mathrm{NMR}\left(\mathrm{CDCl}_{3}, 600 \mathrm{MHz}\right)$ $\delta 9.38$ (s, 2H, H4 and H4'), 9.28 (dd, 2H, $J=4.5,1.8 \mathrm{~Hz}, \mathrm{H} 9$ of phen), 8.26 (dd, 2H, $J=8.1,1.8 \mathrm{~Hz}$, $\mathrm{H} 7$ of phen), 8.21 (d, 2H, $J=7.5 \mathrm{~Hz}, \mathrm{H} 5$ and H5'), 8.01 (s, 2H, H4 of phen), 7.78-7.72 (AB quartet, $4 \mathrm{H}, \mathrm{H} 5$ and $\mathrm{H} 6$ of phen), 7.65 (dd, 2H, $J=8.1,4.5 \mathrm{~Hz}, \mathrm{H} 8$ of phen), 7.43 (td, 2H, $J=7.5,1.5 \mathrm{~Hz}, \mathrm{H} 6$ and $\mathrm{H6}^{\prime}$ ), 7.14 (td, 2H, $J=7.5,1.5 \mathrm{~Hz}, \mathrm{H} 7$ and $\left.\mathrm{H} 7{ }^{\prime}\right), 6.80$ (d, 2H, $J=7.5 \mathrm{~Hz}, \mathrm{H} 8$ and $\left.\mathrm{H} 8^{\prime}\right), 6.68(\mathrm{~s}, 2 \mathrm{H}$, $\mathrm{H} 1$ and $\left.\mathrm{H}^{\prime}\right), 3.12(\mathrm{t}, 4 \mathrm{H}, J=6.3 \mathrm{~Hz}), 2.85(\mathrm{t}, 4 \mathrm{H}, J=6.3 \mathrm{~Hz}) .{ }^{13} \mathrm{C}-\mathrm{NMR}\left(\mathrm{CDCl}_{3}, 62.5 \mathrm{MHz}\right) \delta$ $151156.76,155.83,152.80,151.47,148.89,148.52,141.74,141.46,139.64,136.11,134.46,133.98$, $131.01,128.04,127.89,127.77,124.27,123.86,123.33,122.31,121.50,120.70,119.99,118.83$, 65.97, 28.54, 28.44. MS (ESI): $m / z=725[\mathrm{M}+\mathrm{H}]^{+}$, Anal. Calcd for $\mathrm{C}_{53} \mathrm{H}_{32} \mathrm{~N}_{4} \cdot 0.75 \mathrm{H}_{2} \mathrm{O}: \mathrm{C}, 86.21 ; \mathrm{H}$, 4.57 ; N, 7.59. Found: C, 86.87; H, 4.62; N, 7.65.

\section{Conclusions}

A series of 9,9'-spirobifluorene-derived $N$-heterocycles were prepared from 8,9-dihydrospiro(benzo[b]fluorene-11,9'-fluoren)-6(7H)-one and 8,8',9,9'-tetrahydro-11,11'-spirobi(benzo[b]-fluorene)6,6' $\left(7 H, 7^{\prime} H\right)$-dione and 2-aminoarenecarbaldehydes such as 2-aminobenzaldehyde, 2-aminonicotinealdehyde, 1-amino-2-naphthaldehyde, and 8-aminoquinoline-7-carbaldehyde, respectively. The monoheteroaryl series was crystalline in nature, while diheroaryl ones showed an amorphous nature. In addition to the absorptions based on the parent 9,9'-spirobifluorene skeleton in the 225-234, 239-280, 296-298, and 308-328 nm regions, absorptions due to the $\pi-\pi^{*}$ transition of heterocycles were observed in the 351-375 nm region. All compounds showed strong photoluminescence in the 390-430 $\mathrm{nm}$ region regardless the excitation wavelength.

\section{Supplementary Materials}

Supplementary materials can be accessed at: http:/www.mdpi.com/1420-3049/18/11/13680/s1.

\section{Acknowledgments}

Financial support from National Research Foundation (2010-0012473) is gratefully acknowledged. Jing Lu Liang is a recipient of 2011 postdoctoral fellowship from Yeungnam University. References $11,12,16,17$ were added by referee's recommendation during the revision.

\section{Conflicts of Interest}

The authors declare no conflict of interest. 


\section{References}

1. Clarkson, R.G.; Gomberg, M. Spirans with four aromaatic radicals on the spiro carbon atom. J. Am. Chem. Soc. 1930, 52, 2881-2891.

2. Haas, G.; Prelog, V. Optisch aktive 9,9'-Spirobifluoren-Derivate. Helv. Chim. Acta 1969, 52, 1202-1218.

3. Prelog, V.; Kovačević, M.; Egli, M. Liphophilic tartaric acid esters as enantioselective ionophores. Angew. Chem. Int. Ed. Engl. 1989, 28, 1147-1152.

4. Alcázar Montero, V.; Tomlinson, L.; Houk, K.N.; Diederich, F. Selective $\alpha, \omega$-dicarboxylic acid recognition in a chiral cleft shaped by the 9,9'-spirobifluorene unit. Tetrahedron Lett. 1991, 32, 5309-5312.

5. Alcázar, V.; Diederich, F. Enantioselective complexation of chiral dicarboxylic acids in clefts of functionalized 9,9'-spirobifluorenes. Angew. Chem. Int. Ed. Engl. 1992, 31, 1521-1523.

6. Diederich, F.; Felber, B. Supramolecular chemistry of dendrimers with functional cores. Proc. Natl. Acad. Sci. USA 2002, 99, 4778-4781.

7. Weber, E.; Ahrendt, J.; Czugler, M.; Csöregh, I. Selective inclusion and separation of isomeric homologous hydrocarbons by hydrocarbon host lattices. Angew. Chem. Int. Ed. Engl. 1986, 25, 746-748.

8. Das, G.; Hamilton, A.D. Carbohydrate recognition: Enantioselective spirobifluorene diphosphonate receptors. Tetrahedron Lett. 1997, 38, 3675-3678.

9. Hernández, J.V.; Almaraz, M.; Raposo, C.; Martín, M.; Lithgow, A.; Crego, M.; Caballero, C. Morán, J.R. Chiral recognition of tartaric acid derivatives with chromenone-benzoisoxazole receptors with a spirobifluorene spacer. Tetrahedron Lett. 1998, 39, 7401-7404.

10. Tejeda, A.; Oliva, A.I.; Simón, L.; Grande, M.; Caballero, C.; Morán, J.R. A macrocyclic receptor for the chiral recognition of hydroxycarboxylates. Tetrahedron Lett. 2000, 41, 4563-4566.

11. Poriel, C.; Rault-Berthelot, J.; Thirion, D. Modulation of the electronic properties of $3 \pi$-2spiro compounds derived from bridged oligophenylenes: A structure-property relationship. J. Org. Chem. 2013, 78, 886-898.

12. Huang, J.; Yang, X.; Wang, J.; Zhong, C.; Wang, L.; Qin, J.; Li, Z. New tetraphenylethene-based efficient blue luminophors: Aggregation induced emission and partially controllable emitting color. J. Mater. Chem. 2012, 22, 2478-2484.

13. Müller, C.D.; Falcou, A.; Reckefuss, N.; Rojahn, M.; Wiederhirn, V.; Rudati, P.; Frohne, H.; Nuyken, O.; Becker, H.; Meerholz, K. Multicolor organic light-emitting displays by solution processing. Nature 2003, 421, 829-833.

14. Grimsdale, A.C.; Chan, K.L.; Martin, R.E.; Jokisz, P.G.; Holmes, A.B. Synthesis of light-emitting conjugated polymers for applications in electroluminescent devices. Chem. Rev. 2009, 109, 897-1091.

15. Omer, K.M.; Ku, S.-Y.; Wong, K.-T.; Bard, A.J. Green electrogenerated chemluminescence of highly fluorescent benzothiadiazole and fluorene derivatives. J. Am. Chem. Soc. 2009, 131, 10733-10741.

16. Saragi, T.P.I.; Spehr, T.; Siebert, A.; Fuhrmann-Lieker, T.; Salbeck, J. Spiro compounds for organic optoelectronics. Chem. Rev. 2007, 107, 1011-1065. 
17. Moreau, F.; Audebrand, N.; Poriel, C.; Moizan-Baslé, V.; Ouvry, J. A 9,9'-spirobifluorene based metal-organic framework: Synthesis, structure analysis and gas sorption properties. J. Mater. Chem. 2011, 21, 18715-18722.

18. Ferrand, Y.; Poriel, C.; Le Maux, P.; Rault-Berthelot, J.; Simonneaux, G. Asymmetric heterogeneous carbene transfer catalyzed by optically active ruthenium spirobifluorenylporphyrin polymers. Tetrahedron Asymmetry 2005, 16, 1463-1472.

19. Murase, S.; Tominaga, T.; Kitazawa, D. Organic Electroluminescent Device. Japan Patent 093425, 2005.

20. Wu, F.; Riesgo, E.C.; Thummel, R.P.; Juris, A.; Hissler, M.; Elghayoury, A.; Ziessel, R. Closely-spaced chelating centers: Synthesis of novel spiro-bridged bis-phenathrolines and bis-indole derivatives. Tetrahedron Lett. 1999, 40, 7311-7314.

21. Liao, L.-S.; Begley, W.J.; Pellow, C.A. Phosphorscent OLED having double-blocking layers having different triplet energies. U.S. Patens Appl. Publ. 191427, 2009.

22. Tominaga, T.; Kitazawa, D.; Makiyama, A.; Kohama, A. Light-emitting device material and light-emitting devices. PCT Int. Appl. WO 043449, 2005.

23. Jahng, Y.; Rahman, A.F.M.M. Synthesis and properties of 2,2'-Di(heteroaryl)-9,9'-spirobifluorenes. Bull. Chem. Soc. Jpn. 2010, 83, 672-677.

24. Qu, Y.-W.; Shi, K.-H.; Liu, Q.-C. Synthesis of quinolinyl spirobifluorene derivatives. Chin. J. Synth. Chem. 2008, 16, 15-18.

25. Chea, J.M.; Jahng, Y. Synthesis and properties of 9,9'-spirobifluorene-based heterocycles. Heterocycles 2009, 78, 1573-1580.

26. Opie, J.W.; Smith, L.I. o-Aminobenzaldehyde. Org. Synth. 1955, 56-58.

27. Majewicz, G.; Caluwe, O.A. Facile synthesis of 2-aminonicotinaldehyde. J. Org. Chem. 1974, 39, $720-721$.

28. Riesgo, E.C.; Jin, X.; Thummel, R.P. Introduction of benzo[ $h]$ quinoline and 1,10-phenanthroline subunits by friedländer methodology. J. Org. Chem. 1996, 61, 3017-3022.

29. Thirion, D.; Poriel, C.; Métivier, R.; Rault-Berthelot, J.; Barrière, F.; Jeannin, O. Violet-to-blue tunable emission of aryl-substituted dispirofluorene-indenofluorene isomers by conformationallycontrollable intramolecular excimer formation. Chem. Eur. J. 2011, 17, 10272-10287.

30. Pretsch, E.; Seibl, J.; Simon, W. Tables of Spectral Data for Structural Determination of Organic Compounds, 2nd ed.; Springer-Verlag: Heidelberg, Germany, 1989; H315.

Sample Availability: Samples of the compounds $\mathbf{5 a - d}$ and $7 \mathbf{a}-\mathbf{d}$ are available from the authors.

(C) 2013 by the authors; licensee MDPI, Basel, Switzerland. This article is an open access article distributed under the terms and conditions of the Creative Commons Attribution license (http://creativecommons.org/licenses/by/3.0/). 